\title{
Aggressive periodontitis associated with Kindler syndrome in a large Kindler syndrome pedigree
}

\author{
Tuba Talo Yıldırım¹, Filiz Acun Kaya ${ }^{2}$, Mustafa Taşkesen ${ }^{3}$, Serkan Dündar ${ }^{1}$, \\ Alihan Bozoğlan ${ }^{1}$, Gülücağ Giray Tekin ${ }^{4}$, Sedat Akdeniz ${ }^{5}$

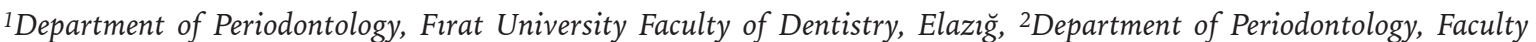 \\ of Dentistry, Departments of ${ }^{3}$ Pediatrics, and ${ }^{5}$ Dermatology, Faculty of Medicine, Dicle University and ${ }^{4}$ Department of \\ Periodontology, Mouth and Dental Health Center, Diyarbakır, Turkey. E-mail: dt_talo@hotmail.com \\ Revised: 19th July 2016, Accepted: 19th January 2017
}

SUMMARY: Talo-Yıldırım T, Acun-Kaya F, Taşkesen M, Dündar S, Bozoğlan A, Tekin GG, Akdeniz S. Aggressive periodontitis associated with Kindler syndrome in a large Kindler syndrome pedigree. Turk J Pediatr 2017; 59: 56-61.

Kindler syndrome (KS) is a rare genetic disorder. The clinical features include aggressive periodontal disease and severe desquamative gingivitis. Five individuals with KS were assessed by oral examination, radiographic analysis and periodontal measurements. All the patients' indexes were recorded prior to periodontal treatment and at the end of the $1^{\text {th }}, 3^{\text {th }}, 6^{\text {th }}, 9^{\text {th }}$ and $12^{\text {th }}$ month respectively. All the patients had improvement of periodontal status and enhancement in index scores. The affected individuals were previously screened for FERMT1 mutations. KS patients' periodontal disease activity could be taken under control with regular follow-up.

Key words: FERMT-1, periodontal disease, periodontal treatment, growth retardation.

Kindler syndrome (KS) is a rare heritable an autosomal recessive disorders characterized by trauma-induced skin blistering, progressive poikiloderma, skin atrophy, mucosal inflammation and varying degrees of photosensitivity ${ }^{1}$. Additional clinical features of $\mathrm{KS}$ are blistering predominantly affecting trauma-prone sites, progressive poikiloderma and skin atrophy especially on the dorsal aspects of the hands and feet ${ }^{1}$. Individuals with KS suffer from photosensitivity which tends to be variably present. The blistering and the photosensitivity often tend to reduce with age ${ }^{2}$.

Other clinical features are different mucosal stenosis that include anal, vaginal, urethral and esophageal stenosis in individuals with KS. Gastrointestinal disorders such as severe colitis and bloody diarrhea, constipation can also occur in patients with $\mathrm{KS}^{2}$.

Oral mucosal manifestations are very common that include glandular cheilitis of the lower lip, angular cheilitis severe periodontal disease (aggressive periodontitis) and severe desquamative gingivitis causing bleeding gums and loss of teeth ${ }^{3,4}$. The association of aggressive periodontitis with KS was first diagnosed in one case in 1996 and later confirmed with a larger population of patients ${ }^{3}$.

The pathogenesis of KS is unclear. Recent studies showed that the FERMT1 gene (Also as known KIND1), is responsible for KS. FERMT1 gene at chromosomal locus is 20p12.3 ${ }^{4}$; where loss-of- function mutations in the FERMT1 have been shown to underlie the molecular basis of this condition. FERMT1 gene encoded is implicated in linking the actin cytoskeleton to the extracellular matrix ${ }^{5}$. In the skin, FERMT1 is mainly expressed in the basal keratinocytes and binds to the cytoplasmic tails of $\beta 1$ and $\beta 3$ integrin's Lack of FERMT1 in keratinocytes would result in decreased cell adhesion, reduced proliferation, increased apoptosis, and loss of cell polarity 5 .

In spite of the expanding FERMT1 mutation database, there seems to be a lack of a clear genotype-phenotype correlation in KS. KS shows extensive clinical heterogeneity in those who have FERMT1 mutations and from within the same ethnic population. Additionally, environmental factors are very important; persons with KS are effected by environmental factors such as oral trauma and sun exposure. 


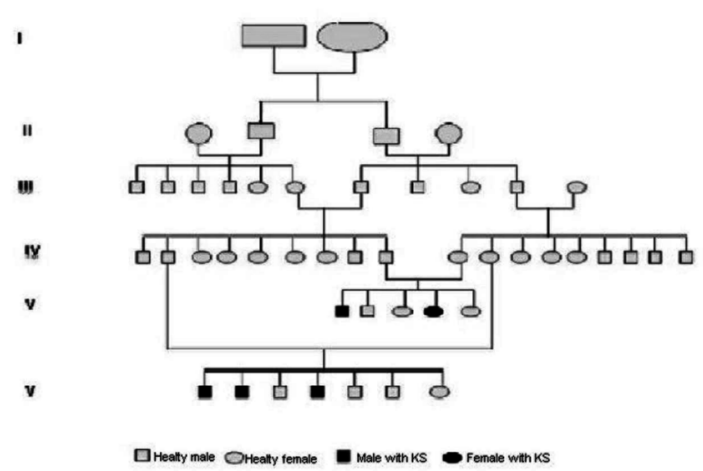

Figure 1: The pedigree of the kindred

Fig. 1. The pedigree of the kindred.

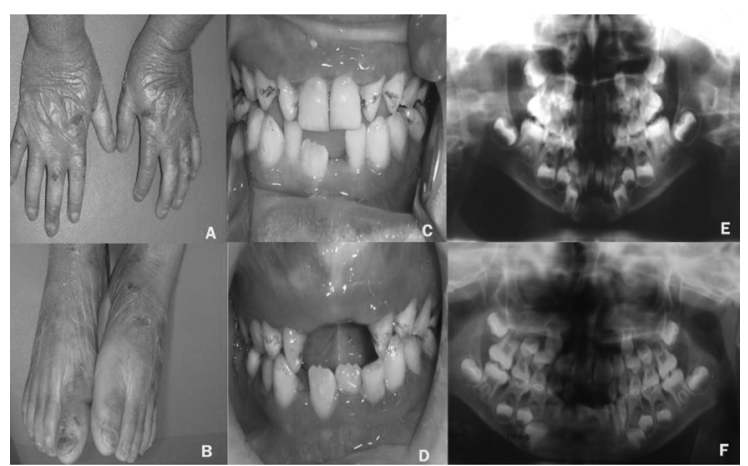

Fig. 2. Clinical features and periodontal status of F/6.

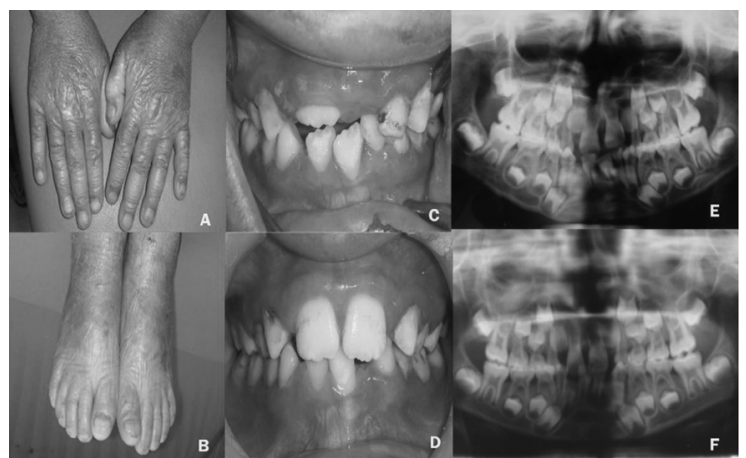

Fig. 3. Clinical features and periodontal status of $M / 7$.

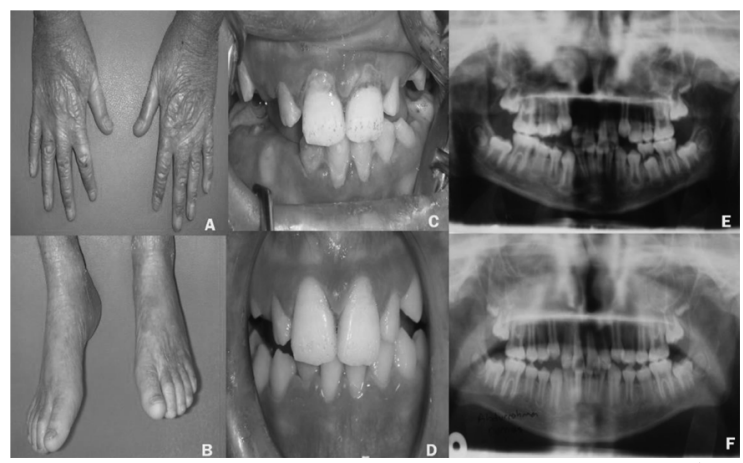

Fig. 4. Clinical features and periodontal status of $M / 10$.

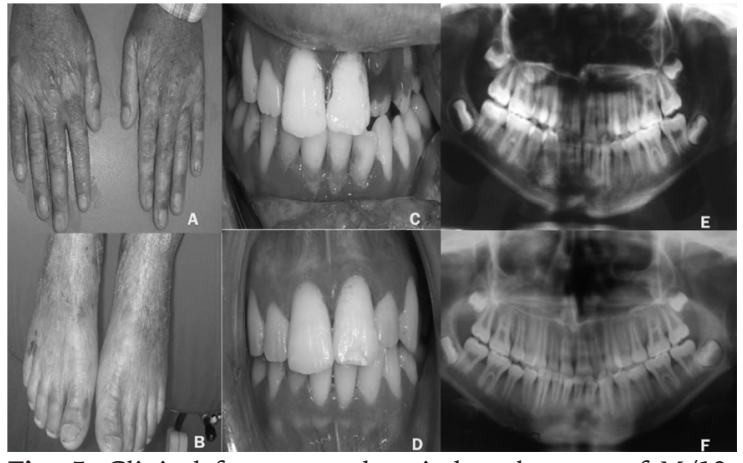

Fig. 5. Clinical features and periodontal status of $M / 12$.

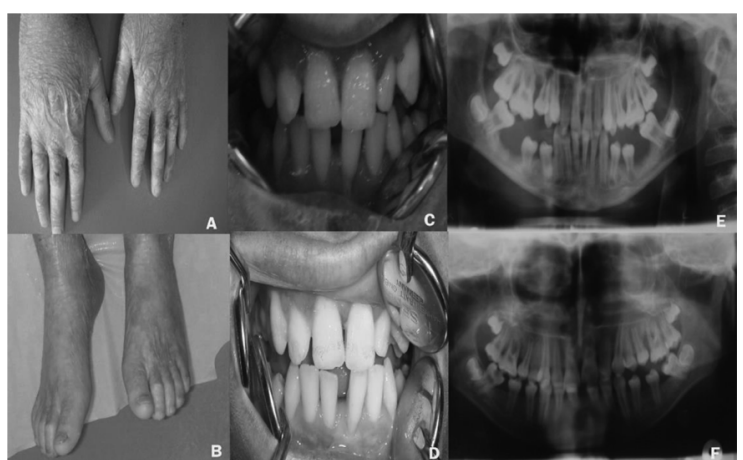

Fig. 6. Clinical features and periodontal status of $\mathrm{M} / 14$.

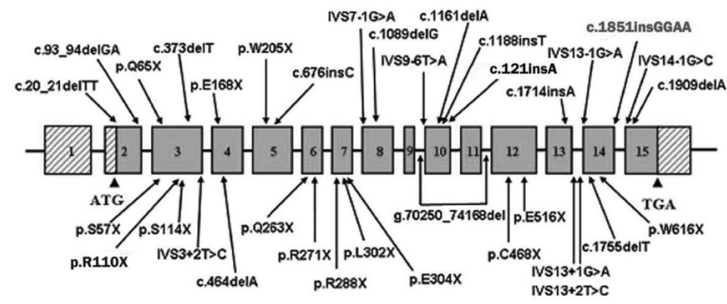

Fig. 7. DNA analysis reveals a homozygous frameshift mutation

Age is an important factor for phenotypic diversity of KS. Patients' clinical characteristic are different with diverse age. Also ethnic and geographical property may influence the clinical heterogeneity of KS. Some studies reported that patient with KS from different geographic place had diverse features previously ${ }^{6}$.

KS is often misdiagnosed and the true prevalance of KS have been reported much higher. To date, over 73 different mutations of the FERMT1 gene have been diagnosed, these consist of: deletions of parts or the entire gene, splice-site mutations, nonsense and frame shift mutations ${ }^{7}$.

Herein, we report the periodontal and gingival features of five related individuals with KS 
Table I. Results of Hand Wrist Graphics and the Cephalometric Analyses.

\begin{tabular}{|c|c|c|c|c|c|}
\hline Parameter & $\mathrm{F} / 6$ & $\mathrm{M} / 7$ & $\mathrm{M} / 10$ & $\mathrm{M} / 12$ & $\mathrm{M} / 14$ \\
\hline Chronologic age & 6 year & 7 year & 10 year & 12 year & 14 year \\
\hline Skeletal age & 4 year 6 month & 5 year 2 month & 8 year 6 month & 10 year 6 month & 11 year 6 month \\
\hline SNA $\left(^{\circ}\right)$ & 79 & 84 & 83 & 81 & 80 \\
\hline SNB $\left(^{\circ}\right)$ & 72 & 76 & 78 & 73 & 77 \\
\hline ANB $\left({ }^{\circ}\right)$ & 7 & 8 & 5 & 8 & 3 \\
\hline $\mathrm{SN} / \mathrm{Go}-\mathrm{Me}\left({ }^{\circ}\right)$ & 40 & 34 & 37 & 37 & 35 \\
\hline Co-A (mm) & 72 & 88 & 83 & 87 & 90 \\
\hline Co-Gn (mm) & 81 & 89 & 105 & 108 & 115 \\
\hline ANS-PNS (mm) & 45 & 56 & 56 & 56 & 54 \\
\hline \multicolumn{2}{|c|}{ Nasolabial angle $\left(^{\circ}\right) 120$} & 112 & 134 & 103 & 128 \\
\hline $\begin{array}{l}\text { Convexity angle } \\
(\mathrm{N}-\mathrm{Sn}-\mathrm{Pog})\left({ }^{\circ}\right)\end{array}$ & 153 & 145 & 154 & 147 & 155 \\
\hline
\end{tabular}

SNA: Sella nasion A point, SNB: Sella nasion B point, ANB: A point nasion B point, SN: sella nasion, Go-Me: Gonion - Menton, Co-A: Condilyon-A point, Co-Gn: Condilyon-Gnathion, ANS-PNS: Anterior nasal spina-Posterior nasal spina, N-Sn-Pog: Nasion Pogonion

harboring the homozygous frameshift mutation, c.1851insGGAA, originally reported by LaiCheong et $\mathrm{al}^{8}$.

\section{Material and Methods}

The present study was conducted to evaluate periodontal health of patients with KS who visited the department of periodontology during the period of 2012 to 2014 .

The pedigree consists of two related families. Family 1 consists of five children, two of whom were affected were a 6 -year old female $[\mathrm{F} / 6]$ and a 14-year old male [M/14]. Family 2 consists of seven children, three of whom were affected were a 7-year old male [M7], a 10-year old male [M/10] and a 12-year old male [M/12]. They were all born to consanguineous parents and have the classical features of acral traumainduced blistering, photosensitivity, mucosal stenosis and poikiloderma. Finger webbing was also seen in all affected individuals. In addition, they suffer with hyperhidrosis, (Figs. $2,3,4,5$, and 6). The cutaneous features pointed to a diagnosis of KS. In accordance to the Declaration of Helsinki Principles, following informed consent, photographs and peripheral blood samples were taken from all the affected individuals and their parents for genetic screening of the FERMT1 gene. Leukocyte genomic DNA was extracted using the QIAamp Blood Midi Kit (Qiagen, Crawley, UK). Polymerase chain reaction amplification of each coding exon and flanking introns of the FERMT1 gene was performed as previously reported. The amplicons were then purified using the Qiagen PCR purification kit. Bidirectional nucleotide sequencing was performed using the Applied Biosystems 3730 DNA Analyser (Applied Biosystems, Warrington, U.K.). DNA analysis revealed a homozygous frameshift mutation, c.1851insGGAA/c.1851insGGAA, in exon 14 of the FERMT1 gene (Fig. 7). The original report of this mutation has already been published.

\section{Results}

Clinical examination included oral examination, radiographic analysis and conventional periodontal measurements. Oral examination revealed a restricted opening of the mouth. $\mathrm{F} / 6, \mathrm{M} / 7$ and $\mathrm{M} / 10$ had mix dentition, $\mathrm{M} / 12$ and $\mathrm{M} / 14$ had completed permanent dentition. By the anamnesis it was determined that the two lower jaw first molars were extracted due to bone loss and gingival regression (M/14).

Radiological examination by panoramic radiography demonstrated light intensity alveolar bone resorption in $\mathrm{F} / 6, \mathrm{M} / 7, \mathrm{M} / 10$, $\mathrm{M} / 12$ and severe alveolar bone resorption in $M / 14$. The patients' cephalometric radiographies were taken and orthodontic measurements were performed. As a result of the cephalometric analyzes, all the patients without $\mathrm{M} / 14$ had a retrognatic mandible and all the patients without M/12 nasolabial angles were increased (Table I). On suspicion that growth rates were retarded, the patients hand wrist radiographies were taken to evaluate their condition. By 
Table II. Periodontal Index Scores.

\begin{tabular}{|c|c|c|c|c|c|}
\hline $\begin{array}{l}\text { Patients } \\
\text { (sex/age) }\end{array}$ & $\begin{array}{l}\text { Index scores } \\
\text { period }\end{array}$ & PI & GI & GBI (\%) & $\mathrm{PD}(\mathrm{mm})$ \\
\hline \multirow[t]{6}{*}{$\mathrm{F} / 6$} & Baseline & 1.77 & 1.47 & 45.6 & 2.12 \\
\hline & First month & 1.24 & 1.15 & 15.2 & 1.80 \\
\hline & Third month & 1.10 & 1.04 & 4.3 & 1.46 \\
\hline & Sixth month & 0.70 & 0.64 & 0 & 1.18 \\
\hline & Nine mouth & 0.64 & 0.55 & 0 & 1.11 \\
\hline & Twelfth month & 0.39 & 0.28 & 0 & 1.02 \\
\hline \multirow[t]{6}{*}{$\mathrm{M} / 7$} & Baseline & 2.13 & 1.58 & 57.5 & 2.83 \\
\hline & First month & 1.69 & 1.39 & 37.5 & 1.91 \\
\hline & Third month & 1.26 & 1.25 & 26.2 & 1.17 \\
\hline & Sixth month & 0.69 & 0.81 & 0 & 1.25 \\
\hline & Nine mouth & 0.60 & 0.43 & 0 & 1.16 \\
\hline & Twelfth month & 0.33 & 0.23 & 0 & 1.01 \\
\hline \multirow[t]{6}{*}{$\mathrm{M} / 10$} & Baseline & 2.60 & 1.80 & 80.5 & 2.90 \\
\hline & First month & 1.61 & 1.36 & 22.2 & 1.62 \\
\hline & Third month & 1.52 & 1.05 & 5.5 & 1.16 \\
\hline & Sixth month & 0.37 & 0.40 & 2.7 & 1.21 \\
\hline & Nine mouth & 0.15 & 0.13 & 0 & 1.14 \\
\hline & Twelfth month & 0.03 & 0.06 & 0 & 1.21 \\
\hline \multirow[t]{6}{*}{$\mathrm{M} / 12$} & Baseline & 2.58 & 1.98 & 84.8 & 2.51 \\
\hline & First month & 1.59 & 1.31 & 33 & 1.89 \\
\hline & Third month & 1.23 & 1.20 & 19.6 & 1.33 \\
\hline & Sixth month & 1 & 1 & 0 & 1.18 \\
\hline & Nine mouth & 0.21 & 0.16 & 0 & 1.13 \\
\hline & Twelfth month & 0 & 0.14 & 0 & 1.13 \\
\hline \multirow[t]{6}{*}{$\mathrm{M} / 14$} & Baseline & 2.07 & 2 & 100 & 2.40 \\
\hline & First month & 2 & 1.80 & 80.5 & 1.84 \\
\hline & Third month & 1.19 & 0.75 & 0 & 1.52 \\
\hline & Sixth month & 0.5 & 0.28 & 0 & 1.35 \\
\hline & Nine mouth & 0.16 & 0.21 & 0 & 1.21 \\
\hline & Twelfth month & 0.02 & 0.02 & 0 & 0.90 \\
\hline
\end{tabular}

PI: Silness-Löe Plaque Index, GI: Löe-Silness Gingival Index, GBI: Ainoma and by Gingival Bleeding Index and PD: Proping Depth

the evaluation it was determined that all the patients had lower bone ages than their chronologic ages. (Table I).

By the periodontal examination severe gingival inflammation was determined; inflammation and periodontal pocket $(>4 \mathrm{~mm})$ in the patients of $\mathrm{F} / 6, \mathrm{M} / 7$ and $\mathrm{M} / 10$, severe inflammation and aggressive periodontitis in $\mathrm{M} / 12$ and $\mathrm{M} / 14$ with deep pockets $(\geq 7 \mathrm{~mm})$. The gingiva was thin and fragile, and the epithelium sloughed with minor abrasion. The gingival margins were rolled and cratered and lifted away from the tooth surface with a gentle blast of air. Before the periodontal treatment all the patients' indexes [Plaque index (PI), Gingival index (GI), Gingival Bleeding Index (GBI) and Probing Depth (PD)] scores were recorded (Table II). Periodontal therapy included scaling and root planning. Oral hygiene instruction was performed and \% 0.2 chlorhexidine mouth rinse was prescribed (two times daily/ 7 days). Patients were treated once a week, for four weeks. At the end of $1^{\text {th }}, 3^{\text {th }}, 6^{\text {th }}, 9^{\text {th }}$ and $12^{\text {th }}$ months, periodontal scores were recorded again. All the patients exhibited healing and enhanced index scores (Table II) and all periodontal scores were comprised. (Table III). By the follow up appointments no tooth was lost and the oral 
hygiene was brought up to optimal levels. It was also determined that $\mathrm{M} / 14$ had caries in left lower jaw second molar. Patient M/14 left lower second molar tooth caries was restorated in pediatric dentistry clinic. All the patients' follow up appointments are being performed once every three months period. Further we will mention the periodontal follow up results.

\section{Discussion}

In our study, we focused on the periodontal features and management of 5 individuals with KS. Although the cutaneous features are well described, little is currently known about the severe oral disease that typifies this condition. In patients with KS, the oral cavity is effected at an early age, with clinical features including angular cheilitis, dental caries and permanent loss of teeth ${ }^{4}$.

$\mathrm{KS}$ is characterized by aggressive periodontitis as well as severe desquamative gingivitis resulting in bleeding gums and loss of teeth ${ }^{6}$. Immune function and inflammatory responses are affected because of systemic disease and weaken barrier defenses can modify the onset and progression of all forms of periodontal diseases. Wiebe et al. ${ }^{9}$ reported that, involving 20 subjects with $\mathrm{KS}$, periodontitis had an early onset (teenage years), and progression of disease was rapid, resembling aggressive periodontitis, compared to patients without KS of same geographic and ethnic background ${ }^{9}$. Commonly, these patients did not receive dental care, and individuals' dental care was poor. Associated factors in developing countries that can badly affect the extent and severity of oral disease in KS include poverty, malnutrition, over-crowding, lack of oral hygiene and irregular dental visit ${ }^{4}$.

The clinical features of periodontal disease in individuals with KS are variable, as is seen for other clinical same types of mutation such as Rothmund-Thomson syndrome and Bloom syndrome ${ }^{6}$. Because many individuals with KS have unreported periodontal status, the variety of periodontal features in KS remains to be explored.

Wiebe et al. ${ }^{9}$ detected no argument of epithelial seal (i.e., junctional epithelium) to the teeth or pocket epithelium in the extracted teeth ${ }^{9}$. On account of this study it can be said; for individuals with KS the first pathologic periodontal conditions begins with bacterial access. Bacterial can gain access to periodontal connective tissue and cause infections like open chronic wounds. Because of its ability to participate in actin filament organization and cell adhesion, kindlin-1 may serve as a key intracellular protein mediating the adhesion of epithelial cells directly to the tooth ${ }^{10}$.

The treatment of KS is symptomatic. In our study, all the patients were given oral hygiene education and conventional periodontal treatments were performed. After treatment, periodontal index scores were decreased significantly by next appointment. With these clinical conclusions it can be said that scaling, root planning and regular dental care are important factors. KS patients' periodontal health may be improved, alveolar bone loss rate can be reduced and patients can use their teeth for a longer time. Regular dental visits are a very important factor to control erosive gingivitis and aggressive periodontitis in patients with KS. Consequently, regular dental care and good oral hygiene is advised for individuals with KS

\section{Conclusion}

In this study, we describe the periodontal features and management of 5 individuals with KS in whom we previously reported a recurrent homozygous KIND1 mutation denoted c. 1851 insGGAA. KS includes aggressive periodontitis and severe desquamative gingivitis. Traditional non-surgical periodontal treatment has beneficial effects for these patients with $\mathrm{KS}$, although teeth with a poor prognosis at initial examination continued to lose support. Due to the clinical results of our study; it can be said that KS patients' periodontal health status can be taken under control, attachment and bone loss can be avoided and teeth could be kept in the mouth for a longer time.

\section{REFERENCES}

1. Kindler T. Congenital poikiloderma with traumatic bulla formation and progressive cutaneous atrophy. Br J Dermatol 1954; 66: 104-111.

2. Petricca G, Leppilampi M, Jiang G, et al. Localization and potential function of kindlin-1 in periodontal tissues. Eur J Oral Sci 2009; 117: 518-527.

3. Wiebe CB, Silver JG, Larjava HS. Early-onset periodontitis associated with Weary-Kindler syndrome: a case report. J Periodontol 1996; 67: 1004-1010. 
4. Krishna CV, Parmar NV, Has C. Kindler syndrome with severe mucosal involvement in childhood. Clin Exp Dermatol 2014; 39: 340-343.

5. Gao Y, Bai JL, Liu XY, et al. A novel large deletion mutation of FERMT1 gene in a Chinese patient with Kindler syndrome. J Zhejiang Univ Sci B 2015; 16: 957-962.

6. Siegel DH, Ashton GH, Penagos HG, et al. Loss of kindlin-1, a human homolog of the Caenorhabditis elegans actin-extracellular-matrix linker protein UNC112, causes Kindler syndrome. Am J Hum Genet 2003; 73: $174-187$.
7. Rognoni E, Ruppert R, Fassler R. The kindlin family: functions, signaling properties and implications for human disease. J Cell Sci 2016; 129: 17-27.

8. Lai-Cheong JE, Tanaka A, Hawche G, et al. Kindler syndrome: a focal adhesion genodermatosis. $\mathrm{Br} \mathrm{J}$ Dermatol 2009; 160: 233-242.

9. Wiebe CB, Petricca G, Hakkinen L, Jiang G, Wu C, Larjava HS. Kindler syndrome and periodontal disease: review of the literature and a 12-year follow-up case. J Periodontol 2008; 79: 961-966.

10. Salonen JI, Kautsky MB, Dale BA. Changes in cell phenotype during regeneration of junctional epithelium of human gingiva in vitro. J Periodontal Res 1989; 24: 370-377. 